\title{
Is Insight Always the Same? A Protocol Analysis of Insight in Compound Remote Associate Problems
}

\author{
Edward A. Cranford ${ }^{1}$ and Jarrod Moss ${ }^{1}$
}

\begin{abstract}
:
Compound Remote Associate (CRA) problems have been used to investigate insight problem solving using both behavioral and neuroimaging techniques. However, it is unclear to what extent CRA problems exhibit characteristics of insight such as impasses and restructuring. CRA problem-solving characteristics were examined in a study in which participants solved CRA problems while providing concurrent verbal protocols. The results show that solutions subjectively judged as insight by participants do exhibit some characteristics of insight. However, the results also show that there are at least two different ways in which people experience insight when solving CRA problems. Sometimes problems are solved and judged as insight when the solution is the first thing considered, but these solutions do not exhibit any characteristics of insight aside from the "Aha!" experience. In other cases, the solution is derived after a longer period of problem solving, and the solution process more closely resembles insight as it is has been traditionally defined in the literature. The results show that separating these two types of solution processes may provide a better understanding of the behavioral and neuroanatomical correlates of insight solutions.
\end{abstract}

\section{Keywords:}

insight, problem solving, restructuring, impasse, compound remote associate problems

\footnotetext{
${ }^{1}$ Mississippi State University

Correspondence concerning this article should be addressed to Jarrod Moss, PO Box 6161, Department of Psychology, Mississippi State University, Mississippi State, MS 39762. Phone: 662-325-8250 Email: jarrod. moss@msstate.edu
} 


\section{Introduction}

Problem solving is an everyday experience that normally enables people to achieve their goals. However, reaching an impasse in problem solving where one does not know how to proceed is common. The experience of insight is associated with suddenly overcoming a prior problem-solving impasse to find a solution (Ohlsson, 1992), but the factors that lead to the phenomenological "Aha!" experience are not fully understood. Insight can be defined by both phenomenological and cognitive features. Phenomenologically, the source of insight solutions is often hard for the problem solver to identify and reaching the solution is associated with an affective response of suddenness and surprise (the "Aha!" Experience). From a problem-solving perspective, insight solutions often occur after an impasse and are obtained through restructuring the representation of a problem (e.g., Bowden, Jung-Beeman, Fleck, \& Kounios, 2005; Ohlsson, 1992; Schooler, Fallshore, \& Fiore, 1995). In other words, the key components of insight are often described as impasse, restructuring, and "Aha!".

Many types of problems have been used to study insight. Classic insight problems have been used extensively, and sometimes the reason for their use is solely based on the fact that they previously have been used to study insight (Weisberg, 1995). With the emergence of neuroimaging techniques (e.g., functional Magnetic Resonance Imaging [fMRI] and Electroencephalography [EEG]), new problems and paradigms have been used to investigate insight (e.g., Bowden et al., 2005). Classic insight problems simply take too long to solve to be useful stimuli for these methodologies. One specific problem type that has been used to examine insight problem solving is the compound remote associate (CRA) problem (Bowden \& Beeman, 1998; Bowden et al., 2005; Bowers, Regehr, Balthazard, \& Parker 1990; Jung-Beemåan et al., 2004; Kounios et al., 2006; Razumnikova, 2007; Subramaniam, Kounios, Parrish, \& Jung-Beeman, 2009; Wiley, 1998). The CRA task, created by Bowden and Jung-Beeman $(1998 ; 2003 \mathrm{~b})$, is a modification of the remote associates task (RAT) created by Mednick (1962). CRA problems involve finding the one word that can form a compound word or phrase with each of three different words. For example, if three words-tree, sauce, and big-are presented, the solution is apple. CRA problems are solved much faster than classic insight problems, and individual CRA problems can be solved by insight or by noninsight search processes (e.g., generate-and-test or trialand-error) regardless of the number of CRA problems that have been solved on previous trials (Bowden \& Jung-Beeman, 2007).

CRA problems are hybrid-type problems because they can be solved through either insight or noninsight processes, as opposed to many classic problems that are pure insight problems in which solving them means an insight occurred (Weisberg, 1995). Bowden and Jung-Beeman (2003a) have found that people can make a subjective distinction between a search, or noninsight, solution to CRA problems and an insight solution process that 
elicits an affective "Aha!" experience. Because of their hybrid nature, CRA problems can be used in neuroimaging studies to examine the neural correlates of insight by subtracting activation associated with noninsight solutions from activation associated with insight solutions (e.g., Cranford \& Moss, 2011; Jung-Beeman et al., 2004; Kounios et al., 2006). Given their use in studies of insight, evaluating the differences between classic insight problems and CRA problems is important for relating results from CRA problem solving to findings using classic insight problems. The present study investigates insight in CRA problem solving through the examination of participants' concurrent verbal protocols recorded during problem-solving efforts.

\section{CRA Problem Solving and Characteristics of Insight}

Schooler et al. (1995) suggested that insight should be understood by what happens rather than what is experienced. In problem solving, the solver moves from an initial state described by the problem statement to a goal state in which a solution has been obtained (Newell \& Simon, 1972). RAT problem solving can be described as a search through knowledge in the form of a problem space (Newell, 1990; Smith, 1995a). Prior studies of CRA problem solving have found that solvers appear to use a generate-and-test strategy in which the solver tries to recall words (e.g., maple) that are associated with one of the problem words (e.g. tree) and then tests if the word can be combined with the other two problem words (Moss, 2006; Moss, Kotovsky, \& Cagan, 2007). Figure 1 shows an example of this behavior in three protocols. If the solver finds that the word does not fit, then he/ she may try to generate other words that are associates of "tree" or, instead, try to find words that go with one of the other two problem words. This method may continue until the solution is found.

Although CRA problems do involve problem-solving processes, it is difficult to assess objectively whether insight actually occurs at solution. Currently, the best way to assess whether the solution to an individual CRA problem occurred with or without insight is to rely on participants' subjective ratings of whether they solved the problem with insight or not (Bowden \& Jung-Beeman, 2007). Rating a solution as insight does not guarantee that impasse and restructuring processes associated with the traditional view of insight truly occurred. However, Bowden and colleagues (2003b; 2005; 2007) claim CRA problems exhibit phenomenological features and components of insight found in classic insight problems and, therefore, can be used to study insight. The characteristics they claim are that the processes leading to solution are often unreportable, the problems misdirect (or fail to direct) retrieval processes, and solvers report an "Aha!" experience. Nevertheless, when a solver reports solving a CRA problem with insight, there should be characteristic evidence of insight-related processes within the problem-solving process or at the very least something which allows for a subjective distinction to be made. 


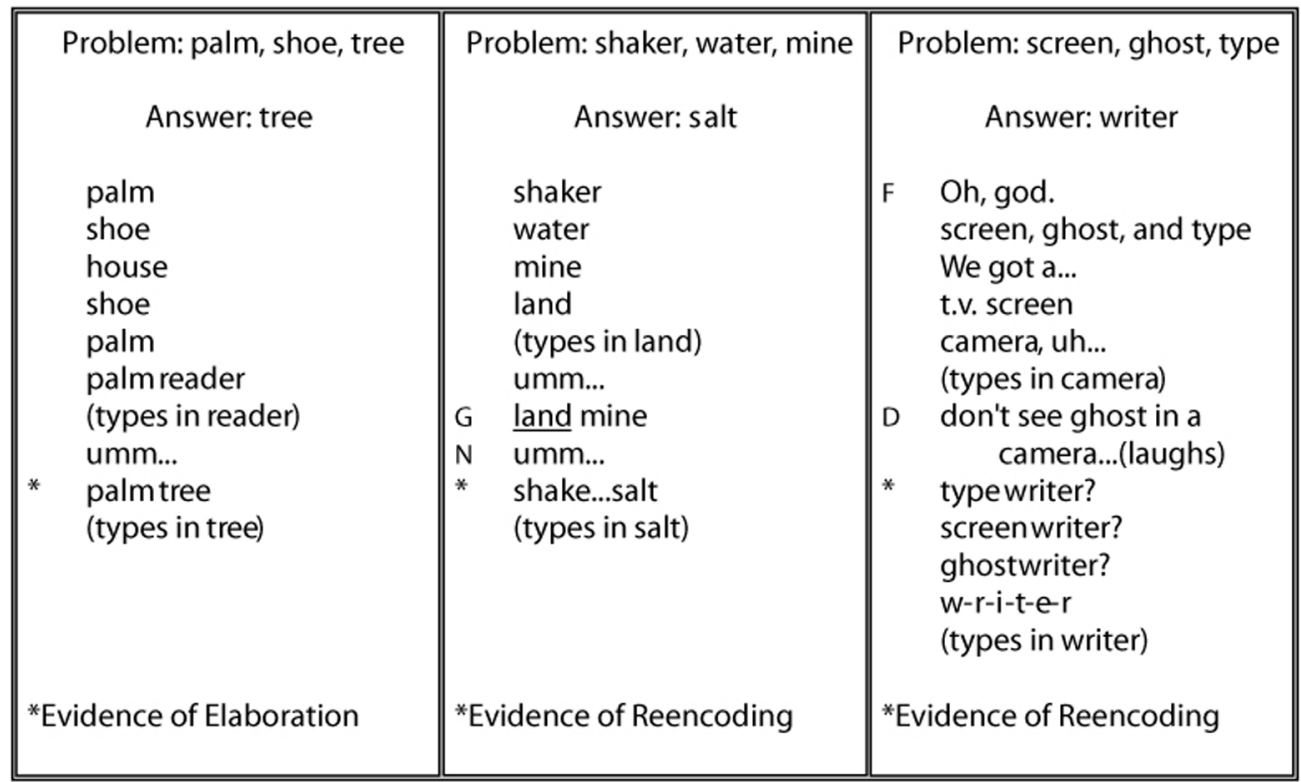

Figure 1. Example verbal protocols of CRA problem solving. The problems on the left and middle are solved with insight and classified as nonimmediate solutions. The problem on the right was solved with noninsight and classified as a nonimmediate solution. Asterisks indicate evidence of Restructuring. Solution candidates are in italics. Repeated solution candidates are underlined. $\mathrm{G}=$ Regenerating Impasse; $\mathrm{N}=$ No new Impasse; F = Frustration Impasse; $D$ = Discontinuing Impasse.

\section{Phenomenological characteristics}

Some research has described insight solely by the problem solvers' unique experience (Gick \& Lockhart, 1995; Kaplan \& Simon, 1990; Schooler et al., 1995). Sometimes referred to as the illumination stage (Smith, 1995b), the "Aha!" experience is the moment a problem solver feels the suddenness of surprise. The experience develops from the sudden emergence of the correct solution to a problem in consciousness (Ohlsson, 1992; Schooler et al., 1995). The "Aha!" experience is often characterized as sudden and surprising, nonconscious, and unintended (i.e., the solver does not intentionally try to solve the problem through insightful processes; Bowden et al., 2005; Ohlsson, 1984; Öllinger, Jones, \& Knoblich, 2008).

When CRA problems are solved, solvers likely base their subjective ratings of whether the problem was insightful or not on this "Aha!" experience. The rating instructions used in prior studies (e.g., Jung-Beeman et al., 2004; Kounios et al., 2006), and the present study, emphasize that decisions of insight be based on the sudden and surprising feeling of an insight that a solver experiences. Therefore, for the purposes of this paper, "insight solutions" are those solutions that were reported as being solved with insight by the participant, which are distinguished from problems reported as being solved without insight (termed "noninsight solutions") by the sudden and surprising "Aha!" experience that the participant feels at solution. If this phenomenological response is the only basis that 
participants use to judge a solution as involving insight, then solutions are categorized as insight solutions and noninsight solutions without relying on any information about whether other component processes of insight were involved in attaining the solution.

The main goal of the research reported in this paper was to examine the characteristics of observable problem-solving behavior that explain whether a solution is subjectively classified as insight or noninsight. In other words, are there characteristics-such as reaching an impasse or restructuring the representation of the problem - that would help to clarify why a problem solver will subjectively classify a problem as insight? A detailed examination of the solution process may reveal that multiple types of problem-solving processes underlie the subjective experience leading to an insight classification for a solved problem. For example, CRA solutions are sometimes the very first candidate word that a person generates. In this case, a solver may report insight simply because the solution was sudden even though it did not require any impasses or restructuring processes. In fact, in the study presented here, two types of problem-solving processes leading to an insight classification are observed: the first type includes solutions in which the first candidate solution generated was the correct solution (these solutions are simply sudden and surprising and, for this paper, are termed "immediate" solutions) and the second type includes solutions in which the first candidate solution was incorrect (these solutions may require both "Aha!" and restructuring and, for this paper, are termed "nonimmediate" solutions). Solutions in which the first candidate generated is correct cannot contain any observable components of insight other than an "Aha!" experience. However, when the solution is not the first candidate generated, there may be other observable components of insight that predict when a solution is classified as insight.

To date, no research has assessed whether solutions rated as being solved with insight are characterized by more observable components of insight than noninsight CRA solutions. Two components of insight that are not discussed in prior CRA insight/noninsight research are impasses and restructuring.

\section{Impasses}

People start to solve a problem with an initial representation of how to solve it. However, the objects, operators, and constraints of a problem produce multiple sources of difficulty, hindering the formation of the correct representation and ultimately the correct answer (Kershaw \& Ohlsson, 2004; Ohlsson, 1992). In an impasse, the solver is either unable to continue with productive problem solving or chooses not to, and an impasse is often a result of an overly constrained representation (Schooler et al., 1995). Either the solver feels he/she has exhausted all available options and can think of nothing else to try or he/she becomes fixated on the wrong objects, relations, or operators. Either way, the impasse is unwarranted in that a solution exists and the solver has the ability to solve it (Ohlsson, 1992). 
Based on Ohlsson's (1992) description of impasse, Fleck and Weisberg (2004) performed a verbal protocol analysis of insight problem solving and produced a list of criteria that represent behaviors typical of impasses (see their Table 2, p.998). The present study uses similar impasse coding criteria for concurrent verbal protocols of CRA problem solving. Sometimes solvers may cease making progress in solving a CRA problem. Three examples of this behavior are evident in CRA problem solving. First, a solver may read the problem words three or more times in succession without generating a solution candidate (termed rereading in our coding scheme). Second, they may completely stop solving the problem altogether without record of any problem-solving behavior (termed discontinuing). Third, a solver may produce no new solution candidates for an extended period of time (termed no-new). Given the short time required to solve many CRA problems, a no-new impasse was coded if a period of 10 seconds elapsed without producing a new candidate solution. Two other behaviors are representative of impasse during CRA problem solving. First, if an incorrect solution is generated two or more times within a problem then this can be seen as a fixation on inappropriate problem elements (termed regenerating). A final criterion for impasse is when a solver exhibited clear signs of emotional frustration and experienced real difficulty with the task or specific problem (termed frustration). Each of the five behaviors is representative of impasse in CRA problem solving. Examples of some coded impasses can be seen in Figure 1.

One source of an impasse may be fixation. Fixation has been defined as the adherence to an inappropriate approach to solving a problem (Smith, 1995b). More generally, fixation can be defined as interference from previous attempts to answer the problem such that it becomes harder to think of new potential solutions. In CRA problem solving, retrieving an inappropriate solution candidate could create a fixation effect by increasing the activation of that item in memory. The high activation of these incorrect solution attempts then makes them the most likely items to be retrieved from memory on future attempts. In the present study, fixation was measured as the number of different candidate solutions generated during problem solving efforts. A smaller number of candidates generated would indicate greater amounts of fixation and more impasses. On the other hand, generating a greater number of candidates indicates less time is spent fixating on inappropriate solutions that lead to impasses. Getting out of an impasse does not necessarily require insight; however, impasses should trigger representational restructuring processes which are the basis for insight (Ohlsson, 1984; 1992). Therefore, greater fixation and more impasses may be more associated with insight solutions than noninsight solutions.

\section{Restructuring}

From the Gestalt perspective, insight occurs when a problem is solved through restructuring, and restructuring can be defined as a change in the solver's representation of the problem, consisting of objects in the problem, relations among the objects, operators that

- volume 4, no. 2 (Spring 2012) 
the solver has available to apply to the objects, and the goal or solution to be obtained (Ash, Jee, \&Wiley, 2012; Weisberg, 1995). Ohlsson (1992) describes three types of restructuring: elaboration, re-encoding, and constraint relaxation. The three types of restructuring defined by Ohlsson were used as coding criteria by Fleck and Weisberg (2004). In order to investigate the frequency of restructuring processes in insight and noninsight solutions to CRA problems, the present study employs a similar coding scheme to analyze the verbal protocols.

When solving a CRA problem, the solver usually tries to retrieve associates related to a single problem word. However, the solution only occasionally has a semantic relation to the base words. Solvers must consider a specific meaning or relation of a problem word in order to retrieve something. For example, consider an attempt to retrieve an associate of the word "star". The associates, "light" or "ship", can be retrieved if the solver considers "star" as a celestial body made of hot gas. If "star" is considered as a celebrity, then the associates, "super" or "movie", can be retrieved. The space of possible candidates that are being examined is defined by the problem words, their currently represented meanings, and the current target word that the subject is using to generate a candidate. These elements define a space in which a memory retrieval operator can generate a candidate solution. A change to one of these elements leads to a different space that can be searched by the retrieval operator. The coding scheme for restructuring used here is based on this analysis of the types of representational change possible in CRA problem solving. Restructuring would involve searching for a change in the problem's representation leading to a change in the problem's search space (e.g., Kaplan \& Simon, 1990). Because restructuring is the basis for insight, there should be a greater proportion of insight solutions containing restructuring than noninsight solutions.

Elaboration means that the current problem representation is extended or enriched in order to move to a representation that leads to a correct solution. Elaboration can occur by noticing elements of the problem that were not initially encoded. Long-term memory may be critical for elaboration because it is a source of additional information. In CRA problem solving, and in the present study, this can be seen when a solver fails to find a solution using one meaning of a problem word and switches to another meaning of the word in order to represent the problem differently. For example, in the problem [star, street, house], if the solver generates candidates based off the word "star", and initially thinks of "star" as a celebrity, then they will not arrive at a solution. However, if a new meaning of the word "star" is considered, such as being a celestial body made of hot gas, then it is possible to arrive at the correct solution, "light". Elaboration is therefore changing the possible space of retrievable candidate solution words by expanding the search space to new meanings of the chosen problem word being used to generate candidate solutions.

Re-encoding involves abandoning one or more components (or interpretations) of the current representation in order to interpret the objects/operators in a new light. 
Through re-encoding the problem solver can form a new representation, see different aspects of the problem, and possibly even apply new operators to these new objects. The goals and prior problem states which were held in working memory fade as a solver abandons a search process in exploration of a different way to represent the problem, possibly leading to the retrieval of new information that leads the solver to a solution (e.g., Langley \& Jones, 1988; Simon, 1977). In the present study, re-encoding is defined as when a solver switches to a different problem word to try and find a solution after failing with a previous word. From the previous example, rather than switching to a new meaning of the word "star", a solver might switch to a new problem word to try and find the solution. Trying to find an associate of "house" may lead to the correct solution. Re-encoding is therefore changing the space of possible solution candidates by abandoning a search of a space defined by the current target problem word and switching to a different target problem word in an attempt to find a solution.

Constraint relaxation occurs as problem solvers relax their ideas of how the objects can be used or relax their ideas of what the problem is asking. Because CRA problems are relatively simple and the instructions and goals are well-defined, it is unlikely that verbal protocols will reveal many, if any, signs of constraint relaxation. Therefore, the present coding scheme has nothing which corresponds to constraint relaxation. Examples of some coded instances of restructuring can be seen in Figure 1.

\section{Using Verbal Protocols to Examine CRA Insight}

The study presented in this paper was designed to examine whether CRA problems solved with a subjective insight experience show objective characteristics of insight problem solving such as impasses and representational restructuring. The results are important because they should help to interpret both prior and future studies using CRA problems to study insight. A verbal protocol analysis of participants' CRA problem-solving efforts was used to determine if CRA problems subjectively rated as solved with insight exhibit more impasses, fixation, and restructuring than problems subjectively rated as solved with noninsight. Secondly, the verbal protocols were used to determine if solutions emerge differently after restructuring for problems judged as insight than problems judged as noninsight. The protocols were also used to determine if quick solutions judged as insight, based only on suddenness of solution (i.e., the first thing thought of), may be different from insights that occur later in problem solving.

The first hypothesis examines whether there are multiple types of problem-solving processes that lead participants to classify a solved problem as insight. It is hypothesized that participants will provide ratings of insight for problems solved when the first candidate generated is correct as well as for problems solved when the first candidate generated

- volume 4, no. 2 (Spring 2012) 
is not correct. The solutions in which the first candidate generated is correct will not be used to examine the second hypothesis.

The second hypothesis assesses to what degree the classic components of insight, obtained from the insight literature, predict whether a participant will rate a CRA problem solution as insight. It is hypothesized that insight solutions should exhibit greater amounts of impasse and fixation than noninsight solutions (Hypothesis 2A-2B). Specifically, there should be more evidence of impasses for problems solved with insight than noninsight (Hypothesis 2A). Also, insight solutions should have greater fixation, as measured by fewer candidates generated for problems solved with insight than noninsight (Hypothesis 2B). The fixation and impasses should lead to an increase in restructuring such that insight solutions contain more evidence of restructuring than noninsight solutions (Hypothesis 2C-2D). Specifically, based on the coding scheme, there should be more evidence of restructuring for problems solved with insight than noninsight (Hypothesis $2 \mathrm{C}$ ).

Also, if insight-producing restructuring processes lead to the solution, then the proportion of problems containing instances of restructuring just prior to solution should be higher for problems solved with insight than problems solved with noninsight (Hypothesis 2D). Ohlsson (1992) hypothesized that, for the insight experience to occur, the change in representation of the problem must bring the goal state within the horizon of the mental look-ahead. In other words, the representation change will trigger insight only when the path to solution is short and quickly obtainable following restructuring. Consistent with Ohlsson's (1992) hypothesis, the presence of restructuring immediately preceding solution may be more associated with insight solutions than noninsight solutions. Insight solutions should therefore have a shorter time to solution after the final restructuring and fewer candidate solutions generated between the final instance of restructuring and solution. For noninsight solutions, after the final restructuring, the solver may still need to spend more time retrieving the solution, contributing to a situation in which the "Aha!" is not experienced. To investigate Hypothesis 2D, the present study examines whether solutions that are generated within a few seconds of restructuring are more likely to be classified as insight than noninsight.

CRA problems may not exhibit evidence supporting all of these hypotheses. However, the hypotheses that are supported should provide some guidance concerning which aspects of insight can be fruitfully studied with CRA problems.

\section{Method}

\section{Participants}

Participants were 31 undergraduates at Mississippi State University who received course credit for their participation. All participants were native English speakers. 


\section{Design}

The design was a 2 (verbal-task: verbalization, nonverbalization) x 3 (solution-type: insight, noninsight, other) within-subject design. Solution-type was measured by the ratings given by the participants, rather than manipulated. The presentation order of verbal-task conditions was counterbalanced across participants.

\section{Materials}

The task, described as word association problems to participants, consisted of a set of 60 CRA problems taken from a larger set of 144 normed CRA items (Bowden \& Jung-Beeman, 2003b). Problems were chosen based on information from a baseline study at Mississippi State University using all 144 problems. The 60 problems with the highest solution rates that had been solved with insight about half of the time were included in the set in order to get a large number of correct solutions per solution-type. An additional six problems were used for practice trials. The problems were presented in random order and randomly assigned to verbal-task condition for each participant. The problems were displayed on a 17-inch computer monitor and answers were given by typing on a keyboard. The task procedure was implemented using E-Prime software (Schneider, Eschman, \& Zuccolotto, 2002). Concurrent verbal protocols were obtained via headset and recorded using E-Prime.

\section{Procedure}

Participants were run individually. After receiving informed consent, participants were instructed on the task and given instructions for classifying a problem's solution as insight, noninsight, or other. These instructions were taken from Jung-Beeman et al. (2004) and Kounios et al. (2006): A feeling of insight is a kind of "Aha!" characterized by suddenness and obviousness. You may not be sure how you came up with the answer, but you are relatively confident that it is correct. The answer seemed to come into your mind all at once ("It just popped into my head"; "Of course!"; "That's it!"; "That's so obvious"). This feeling does not have to be overwhelming, but should resemble what was just described. A noninsight rating is when you strategically searched for the answer by combining possible solutions with each of the three problem words until you felt you had the correct solution. The answer did not just pop into your head, and you felt you had to search for the answer. A rating of 'other' means that you did not know whether the solution was solved with or without insight, you already knew the solution, or you just guessed. The experimenter gave examples of each rating and answered any questions the participants had about the tasks and the rating scale to ensure all instructions were clearly understood. Then the participants read the instructions for a second time presented on the computer. For the verbalization condition, participants were instructed on how to verbalize their thoughts during problem-solving efforts based on the concurrent think-aloud instructions found in the appendix of Ericsson and Simon (1993):

- volume 4, no. 2 (Spring 2012) 
In this experiment we are interested in what you think about when you find answers to some questions that I am going to ask you to answer. In order to do this I am going to ask you to THINK ALOUD as you work on the problem given. What I mean by think aloud is that I want you to tell me EVERYTHING you are thinking from the time you first see the question until you give an answer. I would like you to talk aloud CONSTANTLY from the time I present each problem until you have given your final answer to the question. I don't want you to try to plan out what you say or try to explain to me what you are saying. Just act as if you are alone in the room speaking to yourself. It is most important that you keep talking. If you are silent for any long period of time I will ask you to talk. [Please try to talk clear and loud enough for someone to understand what you are saying.] Do you understand what I want you to do? (p. 378)

After providing this explanation, the experimenter then provided the participant with some practice problems to help them understand how to provide concurrent verbalizations of problem-solving behavior. These think-aloud instructions were used to obtain concurrent verbalizations of problem-solving behavior with minimal interference from problem-solving processes. Participants who completed the verbalization condition first were given instructions and training in how to verbalize their thoughts before the first task and told that they did not have to think aloud anymore right before the second task. For the rest of the participants, think-aloud instructions were given after completing the nonverbalization condition.

Participants were given three practice CRA problems before each verbal-task condition to make sure that they understood the difference in responses for rating a problem (insight, noninsight, and other), were verbalizing (or not) correctly, and understood the task requirements. The practice problems may have also helped to reduce any carryover effects from prior verbalization. After completing the practice trials, participants began the CRA task.

Each CRA problem was presented for a maximum of 30 seconds. The three words making up the problem were presented on separate lines in the middle of the screen. Participants could give a solution at any time during the 30 second interval by typing their answer. If the given solution was incorrect, they could continue work on the problem until the 30-second time limit. Upon solution, participants were prompted to give a rating of whether they solved the problem via insight, noninsight, or other. The order of ratings was counterbalanced so that for half of the participants a rating of 1 was insight and a rating of 3 was noninsight and, for the other half, a rating of 1 was noninsight and a rating of 3 was insight. After a rating was given or the problem remained unsolved for the 30-second time limit, the next problem was presented. Thirty problems were presented and then the participant was asked to stop and notify the experimenter. The experimenter then gave 
the participant the appropriate instructions for the second verbal-task condition; after which, the participant continued to solve the remaining 30 problems.

\section{Data Analysis}

\section{Coding scheme}

The verbal protocols for solved problems were coded for occurrences of impasse and restructuring. The coding categories for each variable (impasse and restructuring) were derived from Fleck and Weisberg (2004). Five types of impasse were coded (rereading, discontinuing, no-new, regenerating, and frustration) and two types of restructuring were coded (elaboration and re-encoding) as described earlier. One clarification about no-new impasses is that they were coded if a participant stopped generating new solution candidates for at least 10 seconds, but all participants were given a standard 5 seconds to read and encode the problem before assessing for a "no-new"impasse. Two independent raters coded the data, and agreement between raters on the number of total impasses (Pearson $r=.84$, Kendall's tau $=.77$ ) and restructuring instances per problem (Pearson $r=.77$, Kendall's tau $=.71$ ) was good. The differences between coders were resolved by having coder 1 re-examine all disagreements. Most disagreements were cases of either coder 1 or 2 missing an instance of impasse or restructuring, and the final version of the data simply included these missed instances. Unfortunately, coder 2 was not available for discussion of the disputes so one of the authors not involved in coding reviewed any remaining disagreements. Although the protocols were coded for all occurrences of impasse and restructuring, sometimes resulting in multiple instances per problem, all analyses examine the presence (1) or absence (0) of impasse and restructuring in each problem.

\section{Mixed effects modeling approach}

The data were analyzed using mixed effects models as opposed to analysis of variance or least squares regression because mixed effects models have a number of advantages over these other approaches (e.g., Baayen, Davidson, \& Bates, 2008; Jaeger, 2008). In particular there are two distinct advantages for this approach with the current data. One is that mixed effects models allow for both participants and problems to be modeled as random effects (as opposed to doing separate by-subject and by-items analyses). The second advantage is that a logistic regression approach can be used (i.e., a logit mixed model) to examine the presence or absence of impasses and restructuring in each problem that participants solved (e.g., Jaeger, 2008). The analyses are reported in regression tables when more than one predictor was included in a model and in-text when only one predictor was used. When more than one factor was used as a predictor in an analysis, the modeling approach used was to include both factors and the interaction between factors in the initial model and then drop the interaction term if it was not significant (a $=.05$ ). Participants and problems were treated as random effects in all analyses.

- volume 4, no. 2 (Spring 2012) 


\section{Verbal overshadowing}

Concurrent verbalization of cognitive processing has been shown to inhibit solving a problem with insight (Schooler, Ohlsson, \& Brooks, 1993). However, other research suggests that differences in verbalization instructions and the nature of the task differentially influence problem solving processes and, by utilizing concurrent verbalization and not requiring the verbalization of visuospatial representations, verbalizing does not necessarily inhibit solutions by insight (Fleck \& Weisberg, 2004; Meissner \& Brigham, 2001). This analysis is not an assessment of whether CRA problems are insight problems, but instead, is meant to assess whether the protocols may be used to examine insight. If verbalization effects are similar for insight and noninsight solutions, then there is evidence that the cognitive processes underlying insight and noninsight solutions are not differentially affected by verbalization and comparisons can be made between insight and noninsight solutions.

In order to examine verbal overshadowing, whether a problem was solved or not was used as a dependent variable with verbal-task as a predictor. The fit of this model is shown in Table 1. There was evidence of verbal overshadowing because the coefficient for verbal-task condition was different from zero reflecting the fact that more problems were solved in the nonverbalization condition than in the verbalization condition. The number of problems solved in each verbal-task condition for each solution-type is shown in Table 2.

In order to examine whether verbalization affected the proportion of solutions classified as insight by participants, solution-type was used as the dependent variable in a mixed logit model with verbal-task condition as the predictor. Due to the low frequency of the "other" solution-type and the fact that only two levels of the dependent variable could be used, the "other" solution-type was excluded from this analysis and all subsequent verbal overshadowing analyses. The second analysis shown in Table 1 shows that verbal-task did not affect the relative proportion of solved problems classified as insight and noninsight. The significance of the intercept in this model is interpretable as reflecting the fact that more solved problems were classified as insight than noninsight.

The impact of verbalization on solution times was analyzed using a mixed effects model with verbal-task and solution-type as predictors presented at the bottom of Table 1. Solution times were longer for noninsight than insight solutions and solution times were also longer for the verbalization than the nonverbalization condition, but there was no interaction. Verbalization decreased the proportion of problems solved and increased solution time but did not differentially affect insight and noninsight solutions.

As an additional check on the impact of verbalization, the data for the participants who were in the nonverbalization condition first were analyzed separately. It could be argued that these participants would be the only ones not affected by verbalization instructions because they did not encounter those instructions until the second half of the study. This group's results did not differ from those reported above, and in general, there 
were no effects of counterbalancing the order of solution-type categories or verbalization task conditions within subjects, so these factors were not included in the reported analyses.

Table 1

Summary of the analyses relevant to verbal overshadowing

\begin{tabular}{lllllll}
\hline Dependent Measure & Predictor & Coefficient & SE & Wald Z & $t$ & $p$ \\
\hline Solved/Not Solved & & & & & & \\
& Intercept & 0.35 & .15 & 2.42 & .02 \\
& Verbal-task & -0.23 & .11 & -2.05 & .04 \\
Insight/Noninsight & & & & & & \\
& Intercept & 0.63 & .19 & 3.35 & .0008 \\
& Verbal-task & 0.08 & .17 & 0.47 & .64 \\
Solution-type (s) & & & & & & \\
& Intercept & 8.19 & .50 & & 16.36 & $<.0001$ \\
& Verbal-task & 1.28 & .45 & & 2.82 & .005 \\
& Solution-type & 4.14 & .49 & & 8.46 & $<.0001$ \\
\hline
\end{tabular}

Note. Verbal-task was coded as 0 for nonverbalization and 1 for verbalization in the coding used to convert factors to regressors. Solution-type was coded as 0 for insight and 1 noninsight. $t$ is reported for models with a continuous dependent measure. Wald $Z$ is reported for logit models with a binary dependent measure.

\section{Results}

Four participants were excluded from all analyses because they reported solving problems only with insight or search but not both. Three participants were excluded because they did not verbalize any solution candidates even after prompting to verbalize.

Participants solved an average of 55\% (SD = 11\%) of problems. For solved problems, the average participant reported solution by insight $56 \%$ (SD $=14 \%$ ) of the time, noninsight $32 \%(S D=16 \%)$ of the time, and other $12 \%(S D=12 \%)$ of the time. The mean solution times and frequencies of these solution categories are shown in Table 2. Because the primary question of interest focuses on insight and noninsight solutions, the other category of solutions were dropped for all analyses, and solution-type (i.e., insight/noninsight) was treated as a categorical dependent variable.

\section{Factors Predicting Insight}

A qualitative examination of the protocols found that many insight solutions had the property that the solution was generated quickly and was the first candidate solution verbalized by the participant. In order to examine this observation quantitatively, solution time and the number of candidates were used as predictors of solution-type (insight 
Table 2

Frequency of solution type and mean solution time for CRA problems

\begin{tabular}{llll}
\hline Solution Type & $\mathrm{N}$ & $\mathrm{M}$ & SD \\
\hline Insight & & & \\
$\quad$ No Think Aloud & 232 & $8.16 \mathrm{~s}$ & $5.63 \mathrm{~s}$ \\
Immediate & 171 & $7.10 \mathrm{~s}$ & $2.73 \mathrm{~s}$ \\
$\quad$ Nonimmediate & 47 & $15.49 \mathrm{~s}$ & $7.25 \mathrm{~s}$ \\
Noninsight & & & \\
$\quad$ No Think Aloud & 132 & $11.56 \mathrm{~s}$ & $7.36 \mathrm{~s}$ \\
$\quad$ Immediate & 47 & $7.84 \mathrm{~s}$ & $3.02 \mathrm{~s}$ \\
$\quad$ Nonimmediate & 71 & $17.64 \mathrm{~s}$ & $5.92 \mathrm{~s}$ \\
Other & & & \\
$\quad$ No Think Aloud & 52 & $11.99 \mathrm{~s}$ & $7.72 \mathrm{~s}$ \\
$\quad$ Immediate & 17 & $8.38 \mathrm{~s}$ & $3.24 \mathrm{~s}$ \\
$\quad$ Nonimmediate & 25 & $18.69 \mathrm{~s}$ & $5.80 \mathrm{~s}$ \\
Unsolved & & & \\
$\quad$ No Think Aloud & 304 & - & - \\
$\quad$ Think Aloud & 342 & - & - \\
\hline
\end{tabular}

coded as 0 , noninsight as 1 ). Solution times for solved problems were measured from the time the problem appeared until the correct solution was entered. This analysis can be seen in the first part of Table 3, where there are effects of the number of candidates, solution time, and an interaction. Consistent with the qualitative observation from the protocols, the effect of the number of candidates shows that the likelihood of a problem being classified as insight decreased as more candidates were generated. Increased solution time was also associated with a lower likelihood of a solution being an insight solution. However, the interaction term shows that increased solution time and an increased number of candidates were associated with a slightly higher likelihood of a problem being classified as insight. These results are consistent with the observation that some problems are classified as insight when the solution if found immediately or soon after starting the problem while other problems are classified as insight after a longer delay between problem presentation and solution.

Based on this analysis and the observation in the protocols, solutions were categorized into two categories of a solution-timing factor. The first type of solution-timing, termed "immediate" occurred when the first candidate solution verbalized was the solution while problems with multiple verbalized candidate solutions were categorized as "nonimmediate". When solution-timing is included in the analysis of solution-type, the number of candidates and the interaction of candidates and solution time are no longer 
significant and the resulting model is shown as the second analysis in Table 3 . In this analysis, solution-timing is the best predictor of solution-type with some significant but reduced effect of solution time.

Table 3

Summary of the analyses of solution-type (insight $=0$ ) for all solved problems

\begin{tabular}{llllll}
\hline Dependent Measure & Predictor & Coefficient & SE & z & $p$ \\
\hline Insight/Noninsight & & & & & \\
& Intercept & -4.27 & 0.64 & -6.70 & $<.001$ \\
& Number Candidates Generated & 0.24 & 0.05 & 5.04 & $<.001$ \\
& Solution Time (s) & 1.49 & 0.42 & 3.57 & $<.001$ \\
& Number Candidates Generated & -.07 & 0.02 & -3.70 & $<.001$ \\
& x Solution Time & & & & \\
Insight/Noninsight & & & & \\
& Intercept & -0.64 & 0.56 & -1.15 & .25 \\
& Solution Time (s) & 0.07 & 0.03 & 2.36 & .02 \\
& Solution-timing & -1.40 & 0.38 & -3.66 & $<.001$ \\
\hline
\end{tabular}

Note. Solution-timing was coded as 0 for nonimmediate solutions and 1 for immediate solutions in the coding used to convert factors to regressors.

A participant may report a quickly solved problem as insight simply because the solution occurred so fast that it seemed sudden and surprising. However, it is unclear whether this type of solution should be grouped together with nonimmediate-insight solutions. It may be that the component cognitive processes in the case of a nonimmediate-insight solution are different from those involved in an immediate-insight solution. In the subsequent analyses, immediate and nonimmediate solutions are separated so that the protocol data can be used to examine what factors predict when a nonimmediate solution will be classified as insight. In other words, the immediate solutions are those where there is a relatively quick solution likely to be classified as insight, and there is correspondingly not much data to analyze in the verbal protocols. Therefore, only nonimmediate solutions are included in subsequent analyses.

However, there were also some solutions in the immediate category where solution time was relatively long (i.e., greater than $15 \mathrm{~s} ; \mathrm{N}=8$ ). These are likely solutions where participants forgot to verbalize during problem solving. We conducted each analysis including all of these solutions as immediate solutions or including these solutions as nonimmediate solutions. The results of these different versions of analyses did not differ, so these solutions were excluded from all subsequent analyses.

- volume 4, no. 2 (Spring 2012) 


\section{Factors Predicting Nonimmediate Insight}

\section{Impasses and fixation}

Impasses were examined using the coding scheme developed for the verbal protocol data to identify problems in which impasses occurred. The range for the number of impasses occurring in a problem was between 0 and 3. The proportion of problems in which impasses occurred is shown in Table 4. Impasses were relatively rare so impasses were examined as being present/absent and only by combining all of the impasse types into a single impasse present/absent variable. Hypothesis $2 \mathrm{~A}$ was not supported because the presence of an impasse was not a significant predictor of whether a nonimmediate solution was classified as insight or noninsight ( $\mid$ Wald $z \mid<1)$.

The fixation analysis examined the number of and rate of unique candidate solutions generated during problem solving. The number of candidates generated and candidate generation rates are sown in Table 4. These measures were used as a metric that should be inversely correlated with fixation. A higher rate of candidate solution generation without repeating previously rejected candidates means that less time is spent in periods where no progress is being made or being spent retrieving previously rejected candidates. There was no difference in the number of candidates generated for nonimmediate-insight and nonimmediate-noninsight solutions (Wald $z=1.42, p=.16$ ). The analysis of generation rates found that nonimmediate-insight solutions had a higher generation rate than nonimmediate-noninsight solutions (Wald $z=-2.095, p=.04$ ), implying that, contrary to Hypothesis $2 \mathrm{~B}$, less fixation may be associated with classifying a problem as being solved by insight.

\section{Restructuring}

Restructuring was examined in two ways. The first analysis used the coding scheme developed for the verbal protocol data to identify problems in which restructuring occurred. The range for the number of restructuring instances that occurred in a problem was between 0 and 3. The proportion of problems in which restructuring occurred is shown in Table 4. Restructuring was relatively rare, so restructuring was examined as being present/ absent and only by combining all of the restructuring types into a single restructuring present/absent variable. Contrary to Hypothesis $2 \mathrm{C}$, the presence of restructuring was not a significant predictor of whether a nonimmediate solution was classified as insight or noninsight ( $\mid$ Wald $z \mid<1)$.

The second analysis for restructuring was done to see if restructuring that occurred soon before a solution was more likely to lead to an insight classification for nonimmediate solutions (Hypothesis 2D). The amount of time between restructuring and solution and the number of solution candidates generated after restructuring but before solution are two different measures of how quickly a problem was solved following restructur- 
Table 4

Mean proportion of nonimmediate solutions in each solution-type containing each impasse type and restructuring type and candidate solution generation rate

\begin{tabular}{lll}
\hline & \multicolumn{2}{l}{ Solution-Type } \\
\cline { 2 - 3 } Predictor & Nonimmediate-Insight & Nonimmediate-Noninsight \\
\hline Any Impasse Present & $.21(.41)$ & $.30(.46)$ \\
$\quad$ Rereading & $.06(.25)$ & $.07(.26)$ \\
Regenerating & $.02(.15)$ & $0(0)$ \\
Discontinuing & $.02(.15)$ & $.06(.23)$ \\
No New & $.11(.31)$ & $.17(.38)$ \\
Frustration & $.11(.31)$ & $.07(.26)$ \\
Any Restructuring Present & $.77(.43)$ & $.63(.49)$ \\
Reencoding & $.72(.45)$ & $.58(.50)$ \\
Elaboration & $.09(.28)$ & $.08(.28)$ \\
Candidates & $2.57(.95)$ & $2.68(1.22)$ \\
Candidate Rate (per s) & $.19(.09)$ & $.16(.06)$ \\
RPS* & $.66(.48)$ & $.44(.50)$ \\
\hline
\end{tabular}

Note. RPS $=$ Restructuring-preceding-solution. ${ }^{*}=$ predicts insight/noninsight classification.

ing. These two metrics were both used in an analysis of the nonimmediate solutions that contained restructuring and the results were suggestive but nonsignificant. Using only nonimmediate problems containing restructuring is required because the number of candidates or amount of time between restructuring and solution is only defined for problems containing any restructuring. Therefore, the two metrics were combined to form a categorical variable which was coded as 1 only for problems where a single candidate (i.e., the solution) was generated within $3 \mathrm{~s}$ of the final occurrence of restructuring in the problem. Three seconds was used because across all problems with any restructuring there was a clear distribution with a long flat tail occurring after $3 \mathrm{~s}$. The choice of whether 2, 3, or $4 \mathrm{~s}$ is used as the cutoff does not influence the result of the analysis. This restructuringpreceding-solution variable was set to 0 for all other nonimmediate solutions (including those without restructuring), and it was used as a predictor of solution-type. In support of Hypothesis 2D, when restructuring preceded a nonimmediate solution the problem was more likely to be classified as insight (Wald $z=-2.04, p=.04$ ).

\section{Discussion}

The purpose of the experiment was to determine if CRA problems that are subjectively classified as being solved with insight exhibit characteristics of insight beyond the "Aha!" 
experience. Participants solved a set of CRA problems while either remaining silent or while providing concurrent verbalization of their problem-solving processes. In order to analyze problem-solving behavior for evidence of insight in problems reported as being solved with insight, the verbal protocols were coded for occurrences of behaviors typical of the classic components of insight: impasse, fixation, and restructuring. To ensure the verbal protocols could be used as data, a verbal overshadowing manipulation check was performed. There was a verbal overshadowing effect for CRA problems resulting in a fewer number of solutions in the verbalization condition, but verbalization did not differentially affect insight and noninsight solutions. Because verbalization did not interact with solution-type, it seems likely that verbalization had similar effects on both noninsight and insight solution processes. The distribution of solution times was very similar for insight and noninsight in both the verbalization and nonverbalization conditions supporting the conclusion that verbalization did not differentially affect problem-solving processes in these conditions. This conclusion is similar to that in other protocol research on insight (Fleck \&Weisberg, 2004). Therefore, the protocol data can be used to investigate the differences between CRA problems reported as being solved with insight and those reported as being solved with noninsight.

The verbal protocol data made it clear that there are multiple problem-solving processes that lead to a solution being classified as insight. The results show that, consistent with Hypothesis 1, there were two apparently different problem-solving processes that participants grouped together into the insight solution category. Immediate solutions were ones in which the correct candidate was the first candidate generated, and these problems were quickly solved and often rated as insight. Nonimmediate solutions were those solutions in which more than one candidate was produced. Immediate-insight solutions had little to no observable characteristics of insight, due to their rapid solution, and may be solutions to problems where the first word that came to mind was the answer. Participants may have reported insight simply because the answer was sudden and resembled an "Aha!". For nonimmediate-insight solutions, the initial candidate retrieved is incorrect and the solution must be obtained through further search or a re-representation of the problem, somehow bringing the solution suddenly into consciousness and invoking an "Aha!" feeling.

Immediate solutions provide no verbal protocol data to analyze. Because the first candidate generated is correct, there is no evidence of impasses or restructuring. The only predictor of insight for immediate solutions is the subjective "Aha!" feeling. Therefore, Hypothesis 2, concerning which components of insight are predictive of classifying a solution as insight, could only be examined for nonimmediate solutions.

The four sub-hypotheses, 2A-2D assessed what, other than a sudden "Aha!" experience, predicts when a solution is classified as insight by examining the presence of behaviors characteristic of insight: impasse, fixation, and restructuring. They stated that 
solutions classified as insight should contain more evidence of impasse, fixation, restructuring, and restructuring-preceding-solution than solutions classified as noninsight. The results for nonimmediate solutions show no differences between insight solutions and noninsight solutions in the proportion of problems containing impasse (Hypothesis $2 A$ ). Hypothesis $2 \mathrm{~B}$ concerns whether problems solved with insight contain more evidence of fixation. Fixation was measured as the number and rate of candidates generated during problem solving. It was hypothesized that participants would generate fewer candidates at a slower rate, indicating greater fixation, for problems solved with insight than problems solved with noninsight. The results for nonimmediate solutions show there was no difference in the number of candidates generated for problems solved with insight and problems solved with noninsight. However, contrary to predictions, the rate of candidates generated was greater for problems solved with insight than problems solved with noninsight. The latter effect is due to the fact that insight solutions were solved faster than noninsight solutions while a similar number of candidates were generated for the two solution categories. It is possible that producing more candidates within a given period of time reduces fixation. It is also possible that getting stuck at an impasse, and resolving it through restructuring quickly, reduces solution time (increasing candidate generation rate) and contributes to the problem being classified as insight (Hypothesis 2D).

The fact that there was very little evidence of impasse in any of the protocols may signify that CRA problems are relatively easy problems and encountering a lengthy impasse may be rare. Impasses, as we have operationally defined them, often require a specified period of time without progress. However, at least in CRA problems, it seems likely that restructuring may be triggered by some difficulty in generating a new candidate solution that does not meet the definition of impasse. The lack of impasses may therefore be a limitation of the coding scheme used in this study. In fact, the result that candidate generation rate is higher for insight problems may imply that many temporary difficulties in generating solutions were overcome quickly without becoming more extended impasses that were captured by the coding scheme. Therefore the analysis of the rate of new candidates may be related to impasses, but candidate generation rate may tap into shorter-term difficulties not classified as impasses.

Although there is little evidence that solvers reach impasses in CRA problem solving, this does not rule out the idea that insight may occur at solution through restructuring. However, contrary to Hypothesis $2 \mathrm{C}$, the results for nonimmediate solutions show no differences between insight solutions and noninsight solutions in the proportion of problems containing restructuring. It seems as though restructuring in the CRA task is a relatively easy process and is a normal part of the CRA problem-solving process, regardless of solution-type. In classic insight problems, such as the classic candle problem used in Fleck \& Weisberg (2004), correct restructuring leads to the solution, and thus insight. Elaboration and reencoding in these types of problems is difficult. Switching to a new 
target problem word, or to a different meaning of the word, in the CRA task is less difficult. Although the presence of restructuring was not a predictor of self-reported insight, the results for nonimmediate solutions do show that restructuring occurring just prior to solution was more associated with insight than noninsight (Hypothesis 2D).

More important than whether restructuring occurred in solution of a CRA problem may be how the solution emerges as the result of the restructuring. Problems in which restructuring was followed immediately by generation of the solution (within $3 \mathrm{~s}$ ) were more likely to be classified as insight than noninsight. Ohlsson (1992) suggests that solutions would be classified as insight if, after restructuring, the solution is quickly and easily attainable. It may be that in some cases restructuring leads to the quick retrieval of a new candidate solution which in turn leads to an "Aha!" experience. In other cases, the restructuring may lead to a more delayed retrieval leading to solution (i.e., noninsight) and a lack of a subjective "Aha!" experience. For example, following restructuring, one or two candidate answers could be retrieved and rejected followed by the retrieval of the answer. This slower solution process following restructuring may then lead to a noninsight classification. With respect to the results showing that there is a greater candidate generation rate for insight solutions, it may be that the extra time spent retrieving one or two incorrect candidate solutions following restructuring leads to the longer solution time (and correspondingly lower candidate generation rate) for noninsight solutions. This reasoning relates the results contradicting Hypothesis $2 \mathrm{~B}$, concerning candidate generation rate, with the results in support of Hypothesis $2 \mathrm{D}$.

Based on these results, it seems that CRA problems may be useful in studying restructuring processes leading to insight in the last few seconds prior to solution. In fact, this finding corresponds well with what has been done in neuroimaging studies in the past leading to the theory that insight may have a greater right temporal lobe contribution due to more coarse-coding in the right hemisphere (Jung-Beeman et al., 2004; Bowden \& Beeman, 1998). The fact that nonimmediate-insight solutions were more similar to nonimmediate-noninsight solutions than they were to immediate-insight solutions means that using the subjective "Aha!" experience as a marker of insight might not be a reliable indicator of impasse and restructuring processes, or at least it indicates that different problem-solving processes can lead to similar subjective insight experiences. It is therefore important to distinguish between these solution processes. Paraphrasing Newell (1973), the effect of averaging over methods "conceals, rather than reveals" (p. 295) any true effect. In fact, given the large proportion of insight solutions falling into the immediate category, our results would have been drastically different had we not made this distinction.

Neuroimaging studies using CRA problems have found more activity in the right anterior superior temporal gyrus, possibly indicating the sudden emergence of the correct solution, that may be facilitated by cognitive control activity prior to problem onset 
in the dorsal anterior cingulate cortex (Jung-Beeman et al., 2004; Kounios et al., 2006; Subramaniam et al., 2009). However, without sub-categorizing insight solutions into those likely to contain and not likely to contain restructuring it is difficult to determine to what extent this activation is indicative of restructuring processes or indicative of other processes tied more to the subject "Aha!" experience. In other words, the activation patterns may represent some processes related to insight but there might be additional areas associated with the restructuring encountered just prior to nonimmediate-insight solutions that were not seen in these past analyses. For example, an immediate-insight solution contains evidence of the "Aha!" experience but not restructuring. Nonimmediate-insight solutions contain evidence of both the "Aha!" experience and restructuring. Therefore, in neuroimaging studies examining insight using CRA problems, subtracting the activation of immediate-insight solutions from nonimmediate-insight solutions may reveal brain regions associated with restructuring while subtracting out processes responsible for the "Aha!" experience. This example is just one benefit coming out of the investigation of the components making up insight solutions to CRA problems.

In a preliminary neuroimaging investigation of CRA problem solving (Cranford \& Moss, 2011), we found nonimmediate-insight and noninsight solutions produced similar patterns of activation, both different from immediate-insight solutions. Nonimmediate-insight solutions did have greater activation in the aforementioned anterior cingulate cortex and right middle temporal gyrus than noninsight solutions suggesting nonimmediateinsight may be a result of heightened activation of words more distantly connected to the problem word, in line with prior findings showing right hemisphere contributions to insight CRA problem solving (Haarmann, George, Smaliy, \& Dien, 2012; Jung-Beeman et al., 2004; Kounios et al., 2006; Subramaniam et al., 2009). However, there were differences between immediate- and nonimmediate-insight solutions that have not been observed before and might be associated with restructuring. A finer-grained analysis of insight solution processes should lead to improved understanding of the neural correlates of insight including the circumstances under which restructuring leads to insight and when it does not.

An alternative interpretation of the null results for Hypotheses $2 \mathrm{~A}$ and $2 \mathrm{C}$ is that the coding criteria used do not adequately capture behaviors indicative of insight and thus are inherently not predictive of insight. For example, reencoding may be just as likely to capture behavior indicative of taking a next step in a systematic search strategy as it is to capture restructuring behavior. However, switching to new problem words or switching to new meanings of the problem words seems to be the only observable measures of representation change in CRA problem solving. Future studies should seek to determine if there are any other possible measures of impasse or restructuring that can be used to predict insight in CRA problem solving, or more generally ascertain what determines when a CRA problem is rated as being solved with insight.

- volume 4, no. 2 (Spring 2012) 
A second limitation of the current study is that verbalization may have affected the results. While the available evidence indicates that verbalization did not differentially affect insight and noninsight solution processes, the possibility can not be completely ruled out. It may be that many of the immediate solutions were actually problems where participants failed to verbalize and that this lack of verbalization led to an increased likelihood of an insight solution classification. One way to address this would be to replicate the current study but instead of relying on protocols to classify solutions as either immediate or nonimmediate, the participant could be asked whether the solution was just the first thing that he/she though of. This method was used in the fMRI study mentioned before (Cranford \& Moss, 2011). It may also be possible to prompt participants to restructure their representation by manipulating a prompt telling them which word in the CRA problem to focus on. The prediction here would be that insight solutions would be more likely to quickly follow these manipulated reencoding prompts than would noninsight solutions.

CRA problems are useful problems to study insight because they can be solved by insight or noninsight processes and are short and simple problems allowing for many trial presentations within a single session. However, because of these facts, CRA problems are different from classic insight problems. The protocols of individual CRA problems reveal much less verbalization than would be seen in most classic insight problems. Many of the CRA problems do not show any evidence of a restructuring and/or impasse. In addition, the types of restructuring and impasses encountered in CRA problem are relatively limited given the well-defined nature of these problems and the limited number of search operators that can be applied. It is unclear whether the types of restructuring observable in these problems are caused by similar cognitive or neural processes as more complex problems. These are limitations that should be addressed in future research on the behavioral and neural basis of restructuring and insight.

In conclusion, CRA problem solutions were found to be classified as being solved with insight when the first candidate solution was generated quickly and was correct as well as when the solution was obtained after more problem solving activity. Therefore, the same phenomenological experience and classification may result from two potentially different problem-solving processes. The nonimmediate-insight solutions seem to resemble classic insight more than the immediate type and are a slightly better fit to the traditional definition of insight because, in the verbal protocols, there was more evidence of restructuring preceding solution for nonimmediate-insight solutions than nonimmediate-noninsight solutions. These results imply that CRA problems could be used to examine these restructuring processes. Hence, future work would benefit from differentiating immediate- and nonimmediate-insight solutions. By pulling apart the two types of insight solutions, the processes of insight can be further explored. 


\section{References}

Ash, I. K., Jee, B. D., \&Wiley, J. (2012). Investigating insight as sudden learning. The Journal of Problem Solving, 4(2), Article 1.

Baayen, R. H., Davidson, D. J., \& Bates, D. M. (2008). Mixed-effects modeling with crossed random effects for subjects and items. Journal of Memory and Language, 59(4), 390412.

Bowden, E. M., \& Beeman, M. J. (1998). Getting the right idea: Semantic activation in the right hemisphere may help solve insight problems. Psychological Science, 9(6), 435440.

Bowden, E.M. \& Jung-Beeman, M. (2003a). Aha! Insight experience correlates with solution activation in the right hemisphere. Psychonomic Bulletin and Review, 10(3), 730-737.

Bowden, E. M., \& Jung-Beeman, M. (2003b). Normative data for 144 compound remote associate problems. Behavioral Research Methods, Instruments, and Computers, 35(4), 634-639.

Bowden, E. M., \& Jung-Beeman, M. (2007). Methods for investigating the neural components of insight. Methods, 42(1), 87-99.

Bowden, E. M., Jung-Beeman, M., Fleck, J., \& Kounios, J. (2005). New approaches to demystifying insight. Trends in Cognitive Sciences, 9(7), 322-328.

Bowers, K. S., Regehr, G., Balthazard, C., \& Parker, K. (1990). Intuition in the context of discovery. Cognitive Psychology, 22(1), 72-110.

Cranford, E. A., \& Moss, J. (2011). An fMRI study of insight using compound remote associate problems. In L. Carlson, C. Hölscher, \& T. Shipley (Eds.), Proceedings of the 33rd Annual Conference of the Cognitive Science Society (pp. 3558-3563). Austin, TX: Cognitive Science Society.

Ericsson, K. A., \& Simon, H. A. (1993). Protocol analysis: Verbal reports as data (Rev. ed.). Cambridge, MA: The MIT Press.

Fleck, J. I., \& Weisberg, R. W. (2004). The use of verbal protocols as data: An analysis of insight in the candle problem. Memory \& Cognition, 32(6), 990-1006.

Gick, M. L., \& Lockhart, R. S. (1995). Cognitive and affective components of insight. In R. J. Sternberg \& J. E. Davidson (Eds.), The nature of insight (pp. 197-228). Cambridge, MA: MIT Press.

Haarmann, H. J., George, T., Smaliy, A. \& Dien, J. (2012). Remote associates test and alpha brain waves. The Journal of Problem Solving, 4(2), Article 4.

Jaeger, T. F. (2008). Categorical data analysis: Away from ANOVAs (transformation or not) and towards logit mixed models. Journal of Memory and Language, 59(4), 434-446.

Jung-Beeman, M., Bowden, E. M., Haberman, J., Frymiare, J. L., Arambel-Liu, S., Greenblatt, R., et al. (2004). Neural activity when people solve verbal problems with insight. PLoS Biology, 2(4), 500-510.

- volume 4, no. 2 (Spring 2012) 
Kaplan, C. A., \& Simon, H. A. (1990). In search of insight. Cognitive Psychology, 22(3), 374-419.

Kershaw, T. C., \& Ohlsson, S. (2004). Multiple causes of difficulty in insight: The case of the nine-dot problem. Journal of Experimental Psychology: Learning, Memory, and Cognition, 30(1), 3-13.

Kounios, J., Frymiare, J. L., Bowden, E. M., Fleck, J. I., Subramaniam, K., Parrish, T. B., et al. (2006). The prepared mind: Neural activity prior to problem presentation predicts subsequent solution by sudden insight. Psychological Science, 17(10), 882-890.

Langley, P., \& Jones, R. (1988). A computational model of scientific insight. In R. J. Sternberg (Ed.), The nature of creativity: Contemporary psychological perspectives (pp. 177-201). New York, NY: Cambridge University Press.

Mednick, S. A. (1962). The associative basis of the creative process. Psychological Review, 69, 220-232.

Meissner, C. A., \& Brigham, J. C. (2001). A meta-analysis of the verbal overshadowing effect in face identification. Applied Cognitive Psychology, 15(6), 603-616.

Moss, J. (2006). The role of open goals in acquiring problem relevant information. Doctoral dissertation, Carnegie Mellon University, Pittsburgh, PA.

Moss, J., Kotovsky, K., \& Cagan, J. (2007). The influence of open goals on the acquisition of problem relevant information. Journal of Experimental Psychology: Learning, Memory, and Cognition, 33(5), 876-891.

Newell, A. (1973). You can't play 20 questions with nature and win. In W. G. Chase (Ed.), Visual Information Processing (pp. 283-308). New York, NY: Academic Press.

Newell, A. (1990). Unified theories of cognition. Cambridge, MA: Harvard University Press.

Newell, A., \& Simon, H. A. (1972). Human problem solving. Oxford, England: Prentice-Hall.

Ohlsson, S. (1984). Restructuring revisited: II. An information processing theory of restructuring and insight. Scandinavian Journal of Psychology, 25(2), 117-129.

Ohlsson, S. (1992). Information-processing explanations of insight and related phenomena. In M. T. Keane \& K. J. Gilhooly (Eds.), Advances in the psychology of thinking (pp. 1-43). London: Harvester Wheatsheaf.

Öllinger, M., Jones, G., \& Knoblich, G. (2008). Investigating the effect of mental set on insight problem solving. Experimental Psychology, 55(4), 269-282.

Razumnikova, O. M. (2007). Creativity related cortex activity in the remote associates task. Brain Research Bulletin, 73(1-3), 96-102.

Schneider, W., Eschman, A., \& Zuccolotto, A. (2002). E-Prime user's guide. Pittsburgh: Psychology Software Tools Inc.

Schooler, J. W., Fallshore, M., \& Fiore, S. M. (1995). Epilogue: Putting insight into perspective. In R. J. Sternberg \& J. E. Davidson (Eds.), The nature of insight (pp. 559-587). Cambridge, MA: MIT Press.

Schooler, J. W., Ohlsson, S., \& Brooks, K. (1993). Thoughts beyond words: When language overshadows insight. Journal of Experimental Psychology: General, 122(2), 166-183. 
Simon, H. A. (1977). Models of discovery and other topics in the methods of science. Dordrecht, Holland: Reidel.

Smith, S. M. (1995a). Fixation, incubation, and insight in memory and creative thinking. In S. M. Smith, T. B. Ward, \& R. A. Finke (Eds.), The creative cognition approach (pp. 135156). Cambridge, MA, US: The MIT Press.

Smith, S. M. (1995b). Getting into and out of mental ruts: A theory of fixation, incubation, and insight. In R. J. Sternberg \& J. E. Davidson (Eds.), The nature of insight (pp. 229251). Cambridge, MA: MIT Press.

Subramaniam, K., Kounios, J., Parrish, T. B., \& Jung-Beeman, M. (2009). A brain mechanism for facilitation of insight by positive affect. Journal of Cognitive Neuroscience, 21(3), 415-432.

Weisberg, R.W. (1995). Prolegomena to theories of insight in problem solving: A taxonomy of problems. In R. J. Sternberg \& J. E. Davidson (Eds.), The nature of insight (pp. 157196). Cambridge, MA: MIT Press.

Wiley, J. (1998). Expertise as mental set: The effects of domain knowledge in creative problem solving. Memory \& Cognition, 26(4), 716-730.

\section{Acknowledgments}

Some of the results were presented at the 32nd Annual Conference of the Cognitive Science Society. We would like to thank Andrew Watkins, Willie Sullivan, Ariel Sibley, Bailey Barham, Alesha Lindsey, and especially Joshua Liddell for their help with data collection, analysis, and transcription.

- volume 4, no. 2 (Spring 2012) 